\title{
Equivalence of Euler equations and torque-angular momentum relation
}

\author{
V. Tanrıverdi \\ tanriverdivedat@googlemail.com
}

Received 26 June 2020; accepted 18 November 2020

Euler derived equations for rigid body rotations in the body reference frame and the stationary reference frame by considering an infinitesimal part of the rigid body. Another derivation is possible, and it is widely used: transforming torque-angular momentum relation to the body reference frame. However, their equivalence is not shown explicitly. In this work, for a rigid body with different moments of inertia, we calculated Euler equations explicitly in the body reference frame and the stationary reference frame and torque-angular momentum relation. We also calculated equations of motion from Lagrangian. These calculations show that all four of them are equivalent.

Keywords: Rigid body; rotational motion; Euler equations; torque-angular momentum relation.

PACS: 45.40.-f

DOI: https://doi.org/10.31349/RevMexFisE.18.136

\section{Introduction}

Euler derived governing equations for rigid body rotations firstly in the stationary reference frame and then in the body reference frame $[1,2]$. Well-known Euler equations describe rotations of rigid bodies in the body reference frame, and these are used to study rotations of rigid bodies. In recent work, Euler's derivations are given $[3,4]$.

There is an alternative way to Euler's derivation. One can write the time derivative of the angular momentum vector in the body reference frame, and then one can obtain Euler equations from torque-angular momentum relation, $\vec{\tau}=$ $d \vec{L} / d t$, by using $(d \vec{L} / d t)_{S^{\prime}}=(d \vec{L} / d t)_{S}+\vec{w} \times \vec{L}$. This derivation or an equivalent one is used in various works [5-23]. Differently from these, Lamb, Crabtree, and Hayward follow a derivation similar to Euler's derivation to get Euler equations [24-26].

Euler equations in the stationary reference frame have a complicated structure due to changing moments of inertia and inertia products, and they are rarely used and not known widely. Klein and Sommerfeld used Euler's equations in the stationary reference frame [27]. Leimanis mentioned Euler's derivation; however, he did not mention Euler's derivation in the stationary reference frame [22]. Most of the books on classical mechanics or rotations of rigid bodies do not mention them [5-21].

Theoretically, one can expect that Euler's derivations in both reference frames are consistent. A similar expectation is also valid for torque-angular momentum relation. However, their equivalence is not explicitly shown previously in the author's knowledge. In this work, we will explicitly calculate Euler equations in both reference frames and torque-angular momentum relation by using Euler angles, and these calculations will show that they are explicitly equivalent. In the appendix, another set of equivalent equations will be obtained from Lagrangian.

\section{Explicit calculations by using Euler angles}

We will use Euler angles and their time derivatives to calculate Euler equations in both reference frames and torqueangular momentum relation explicitly. Euler angles can be seen in Fig. 1 together with rotation directions, coordinates of the body, and stationary reference frames. In the figure, the rigid body is represented by a stone to emphasize that it should be rigid, and it can have any shape, which results in different magnitudes for moments of inertia. Its mass density can also change with any coordinate variable. Line of nodes, $N$, can also be seen in the same figure, which corresponds to intersection points of $x^{\prime} y^{\prime}$-plane and $x y$-plane. $\dot{\theta}, \dot{\phi}$ and $\dot{\psi}$

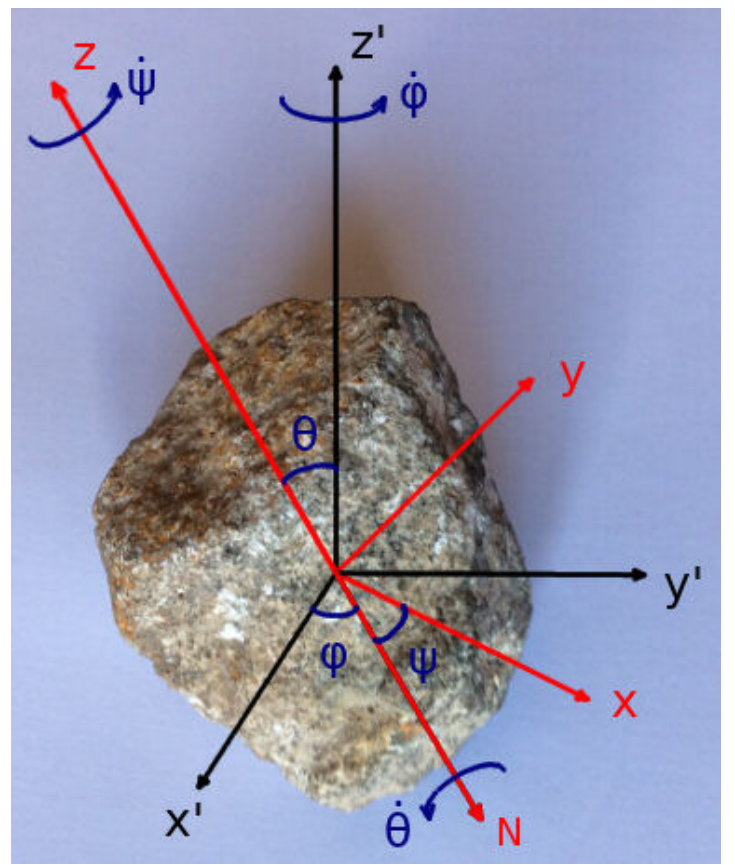

FIGURE 1. Stationary reference frame $\left(x^{\prime}, y^{\prime}, z^{\prime}\right)$, body reference frame $(x, y, z)$, line of nodes $N$, Euler angles $(\theta, \phi, \psi)$, and angular velocities $(\dot{\theta}, \dot{\phi}, \dot{\psi})$. 
define rotations around line of nodes, stationary $z$-axis, and body $z$-axis, respectively. All rotations of rigid bodies can be described by using these rotations.

\subsection{Euler equations}

In this section, we will calculate Euler equations in the body reference frame in terms of Euler angles and their time derivatives.

In the body reference frame, whose axes are chosen as principal axes of inertia tensor, the moment of inertia tensor for a rigid body can be written as

$$
I_{b}=\left[\begin{array}{ccc}
I_{x} & 0 & 0 \\
0 & I_{y} & 0 \\
0 & 0 & I_{z}
\end{array}\right]
$$

where $I_{x}, I_{y}$, and $I_{z}$ are moments of inertia along principal axes. For such a rigid body, Euler equations are [5]

$$
\begin{aligned}
\tau_{x} & =I_{x} \dot{w}_{x}-w_{y} w_{z}\left(I_{y}-I_{z}\right), \\
\tau_{y} & =I_{y} \dot{w}_{y}-w_{z} w_{x}\left(I_{z}-I_{x}\right), \\
\tau_{z} & =I_{z} \dot{w}_{z}-w_{x} w_{y}\left(I_{x}-I_{y}\right),
\end{aligned}
$$

where $\tau_{i}$ 's are components of torque along axes of the body reference frame. Components of the angular velocity can be written by using Euler angles as

$$
\begin{aligned}
& w_{x}=\dot{\phi} \sin \theta \sin \psi+\dot{\theta} \cos \psi, \\
& w_{y}=\dot{\phi} \sin \theta \cos \psi-\dot{\theta} \sin \psi, \\
& w_{z}=\dot{\phi} \cos \theta+\dot{\psi} .
\end{aligned}
$$

Then by using Euler angles, Euler equations can be written explicitly as

$$
\begin{aligned}
\tau_{x} & =I_{x} \ddot{\phi} \sin \theta \sin \psi+I_{x} \ddot{\theta} \cos \psi+\dot{\theta} \dot{\phi} \cos \theta \sin \psi\left(I_{x}+I_{y}-I_{z}\right) \\
& +\dot{\theta} \dot{\psi} \sin \psi\left(I_{y}-I_{x}-I_{z}\right)+\dot{\phi} \dot{\psi} \sin \theta \cos \psi\left(I_{x}+I_{z}-I_{y}\right)+\dot{\phi}^{2} \sin \theta \cos \theta \cos \psi\left(I_{z}-I_{y}\right), \\
\tau_{y} & =I_{y} \ddot{\phi} \sin \theta \cos \psi-I_{y} \ddot{\theta} \sin \psi+\dot{\theta} \dot{\phi} \cos \theta \cos \psi\left(I_{x}+I_{y}-I_{z}\right) \\
& +\dot{\theta} \dot{\psi} \cos \psi\left(I_{x}-I_{y}-I_{z}\right)+\dot{\phi} \dot{\psi} \sin \theta \sin \psi\left(I_{x}-I_{y}-I_{z}\right)+\dot{\phi}^{2} \sin \theta \cos \theta \sin \psi\left(I_{x}-I_{z}\right), \\
\tau_{z} & =I_{z} \ddot{\phi} \cos \theta+I_{z} \ddot{\psi}+\dot{\theta} \dot{\phi} \sin \theta\left[\left(I_{y}-I_{x}\right)\left(\cos ^{2} \psi-\sin ^{2} \psi\right)-I_{z}\right] \\
& +\dot{\theta}^{2} \sin \psi \cos \psi\left(I_{x}-I_{y}\right)+\dot{\phi}^{2} \sin ^{2} \theta \sin \psi \cos \psi\left(I_{y}-I_{x}\right) .
\end{aligned}
$$

These equations describe the change in rotation of a rigid body under the influence of torque in the body reference frame.

\subsection{Euler equations in the stationary reference frame}

In this part, we will explicitly calculate Euler equations in the stationary reference frame by using Euler angles.

Euler also derived equations in the stationary reference frame [1], and it can be written as [4]

$$
\begin{aligned}
& \tau_{x^{\prime}}=I_{x^{\prime} x^{\prime}} \dot{w}_{x^{\prime}}+I_{x^{\prime} y^{\prime}} \dot{w}_{y^{\prime}}+I_{x^{\prime} z^{\prime}} \dot{w}_{z^{\prime}}-I_{x^{\prime} y^{\prime}} w_{x^{\prime}} w_{z^{\prime}}+I_{x^{\prime} z^{\prime}} w_{x^{\prime}} w_{y^{\prime}}+I_{y^{\prime} z^{\prime}}\left(w_{y^{\prime}}^{2}-w_{z^{\prime}}^{2}\right)+w_{y^{\prime}} w_{z^{\prime}}\left(I_{z^{\prime} z^{\prime}}-I_{y^{\prime} y^{\prime}}\right), \\
& \tau_{y^{\prime}}=I_{y^{\prime} y^{\prime}} \dot{w}_{y^{\prime}}+I_{x^{\prime} y^{\prime}} \dot{w}_{x^{\prime}}+I_{y^{\prime} z^{\prime}} \dot{w}_{z^{\prime}}-I_{y^{\prime} z^{\prime}} w_{x^{\prime}} w_{y^{\prime}}+I_{x^{\prime} y^{\prime}} w_{y^{\prime}} w_{z^{\prime}}+I_{x^{\prime} z^{\prime}}\left(w_{z^{\prime}}^{2}-w_{x^{\prime}}^{2}\right)+w_{x^{\prime}} w_{z^{\prime}}\left(I_{x^{\prime} x^{\prime}}-I_{z^{\prime} z^{\prime}}\right), \\
& \tau_{z^{\prime}}=I_{z^{\prime} z^{\prime}} \dot{w}_{z^{\prime}}+I_{x^{\prime} z^{\prime}} \dot{w}_{x^{\prime}}+I_{y^{\prime} z^{\prime}} \dot{w}_{y^{\prime}}-I_{x^{\prime} z^{\prime}} w_{y^{\prime}} w_{z^{\prime}}+I_{y^{\prime} z^{\prime}} w_{x^{\prime}} w_{z^{\prime}}+I_{x^{\prime} y^{\prime}}\left(w_{x^{\prime}}^{2}-w_{y^{\prime}}^{2}\right)+w_{x^{\prime}} w_{y^{\prime}}\left(I_{y^{\prime} y^{\prime}}-I_{x^{\prime} x^{\prime}}\right) .
\end{aligned}
$$

To calculate Eqs. (7) explicitly, we need the moment of inertia tensor in the stationary reference frame. We will get it by using the transformation matrix from the body reference frame to the stationary reference frame. This transformation matrix can be obtained in terms of Euler angles as [5]

$$
S=\left[\begin{array}{ccc}
\cos \phi \cos \psi-\cos \theta \sin \phi \sin \psi & -\cos \phi \sin \psi-\cos \theta \sin \phi \cos \psi & \sin \theta \sin \phi \\
\sin \phi \cos \psi+\cos \theta \cos \phi \sin \psi & -\sin \phi \sin \psi+\cos \theta \cos \phi \cos \psi & -\sin \theta \cos \phi \\
\sin \theta \sin \psi & \sin \theta \cos \psi & \cos \theta
\end{array}\right]
$$


The inverse of $S$ can be obtained by using $S_{i j}^{-1}=S_{j i}$.

The moment of inertia tensor in the stationary reference frame can be obtained by using relation $I=S I_{b} S^{-1}[5]$ as

$$
I=\left[\begin{array}{ccc}
I_{x^{\prime} x^{\prime}} & I_{x^{\prime} y^{\prime}} & I_{x^{\prime} z^{\prime}} \\
I_{y^{\prime} x^{\prime}} & I_{y^{\prime} y^{\prime}} & I_{y^{\prime} z^{\prime}} \\
I_{z^{\prime} x^{\prime}} & I_{z^{\prime} y^{\prime}} & I_{z^{\prime} z^{\prime}}
\end{array}\right],
$$

where moments of inertia are given by

$$
\begin{aligned}
I_{x^{\prime} x^{\prime}} & =\left(I_{y}-I_{x}\right) 2 \cos \theta \sin \phi \cos \phi \sin \psi \cos \psi+\cos ^{2} \phi\left(I_{x} \cos ^{2} \psi+I_{y} \sin ^{2} \psi\right) \\
& +\cos ^{2} \theta \sin ^{2} \phi\left(I_{x} \sin ^{2} \psi+I_{y} \cos ^{2} \psi\right)+I_{z} \sin ^{2} \theta \sin ^{2} \phi \\
I_{y^{\prime} y^{\prime}} & =\left(I_{x}-I_{y}\right) 2 \cos \theta \sin \phi \cos \phi \sin \psi \cos \psi+\sin ^{2} \phi\left(I_{x} \cos ^{2} \psi+I_{y} \sin ^{2} \psi\right) \\
& +\cos ^{2} \theta \cos ^{2} \phi\left(I_{x} \sin ^{2} \psi+I_{y} \cos ^{2} \psi\right)+I_{z} \sin ^{2} \theta \cos ^{2} \phi \\
I_{z^{\prime} z^{\prime}} & =\sin ^{2} \theta\left(I_{x} \sin ^{2} \psi+I_{y} \cos ^{2} \psi\right)+I_{z} \cos ^{2} \theta
\end{aligned}
$$

and inertia products are given by

$$
\begin{aligned}
I_{x^{\prime} y^{\prime}} & =I_{y^{\prime} x^{\prime}}=\left(I_{x}-I_{y}\right)\left(\cos ^{2} \phi-\sin ^{2} \phi\right) \cos \theta \sin \psi \cos \psi \\
& +\sin \phi \cos \phi\left[I_{x} \cos ^{2} \psi+I_{y} \sin ^{2} \psi-\cos ^{2} \theta\left(I_{x} \sin ^{2} \psi+I_{y} \cos ^{2} \psi\right)-I_{z} \sin ^{2} \theta\right], \\
I_{x^{\prime} z^{\prime}} & =I_{z^{\prime} x^{\prime}}=\sin \theta\left[\left(I_{x}-I_{y}\right) \cos \phi \sin \psi \cos \psi+\cos \theta \sin \phi\left(I_{z}-I_{x} \sin ^{2} \psi-I_{y} \cos ^{2} \psi\right)\right], \\
I_{y^{\prime} z^{\prime}} & =I_{z^{\prime} y^{\prime}}=\sin \theta\left[\left(I_{x}-I_{y}\right) \sin \phi \sin \psi \cos \psi+\cos \theta \cos \phi\left(I_{x} \sin ^{2} \psi+I_{y} \cos ^{2} \psi-I_{z}\right)\right] .
\end{aligned}
$$

Since $\theta, \phi$, and $\psi$ change with time, components of the moment of inertia tensor also change with time. This is the reason that lets Euler derive equations in the body reference frame.

The angular velocities in the stationary reference frame can be written in terms of Euler angles as [5]

$$
\begin{aligned}
& w_{x^{\prime}}=\dot{\theta} \cos \phi+\dot{\psi} \sin \theta \sin \phi, \\
& w_{y^{\prime}}=\dot{\theta} \sin \phi-\dot{\psi} \sin \theta \cos \phi, \\
& w_{z^{\prime}}=\dot{\phi}+\dot{\psi} \cos \theta
\end{aligned}
$$

Then by using Euler angles and components of the moment of inertia tensor, Eqs. (7) can be obtained as

$$
\begin{aligned}
\tau_{x^{\prime}} & =\ddot{\theta}\left[\left(I_{y}-I_{x}\right) \cos \theta \sin \phi \sin \psi \cos \psi+\left(I_{x} \cos ^{2} \psi+I_{y} \sin ^{2} \psi\right) \cos \phi\right]+\ddot{\psi} I_{z} \sin \theta \sin \phi \\
& +\ddot{\phi}\left[\left(I_{x}-I_{y}\right) \sin \theta \cos \phi \sin \psi \cos \psi+\left(I_{z}-I_{x} \sin ^{2} \psi-I_{y} \cos ^{2} \psi\right) \sin \theta \cos \theta \sin \phi\right] \\
& +\dot{\theta} \dot{\phi} \sin \phi\left[-I_{x} \cos ^{2} \psi-I_{y} \sin ^{2} \psi+\left(I_{z}-I_{x} \sin ^{2} \psi-I_{y} \cos ^{2} \psi\right)\left(\cos ^{2} \theta-\sin ^{2} \theta\right)\right] \\
& +\dot{\theta} \dot{\psi}\left\{\left(I_{y}-I_{x}\right) 2 \cos \phi \sin \psi \cos \psi+\left[I_{z}+\left(I_{x}-I_{y}\right)\left(\sin ^{2} \psi-\cos ^{2} \psi\right)\right] \cos \theta \sin \phi\right\} \\
& +\dot{\phi} \dot{\psi} \sin \theta\left\{\left(I_{y}-I_{x}\right) 2 \cos \theta \sin \phi \sin \psi \cos \psi+\left[I_{z}+\left(I_{x}-I_{y}\right)\left(\cos ^{2} \psi-\sin ^{2} \psi\right)\right] \cos \phi\right\} \\
& +\dot{\phi}^{2} \sin \theta\left[\left(I_{y}-I_{x}\right) \sin \phi \sin \psi \cos \psi+\left(I_{z}-I_{x} \sin ^{2} \psi-I_{y} \cos ^{2} \psi\right) \cos \theta \cos \phi\right] \\
& +\dot{\theta}^{2}\left(I_{x}-I_{y}\right) \sin \theta \sin \phi \sin \psi \cos \psi, \\
\tau_{y^{\prime}} & =\ddot{\theta}\left[\left(I_{x}-I_{y}\right) \cos \theta \cos \phi \sin \psi \cos \psi+\left(I_{x} \cos ^{2} \psi+I_{y} \sin ^{2} \psi\right) \sin ^{2}\right]-\ddot{\psi} I_{z} \sin \theta \cos \phi \\
& +\ddot{\phi} \sin \theta\left[\left(I_{x}-I_{y}\right) \sin \phi \sin \psi \cos \psi+\left(I_{x} \sin ^{2} \psi+I_{y} \cos ^{2} \psi-I_{z}\right) \cos \theta \cos \phi\right] \\
& +\dot{\theta} \dot{\phi}\left[\left(I_{x} \cos { }^{2} \psi+I_{y} \sin { }^{2} \psi\right) \cos \phi+\left(I_{x} \sin ^{2} \psi+I_{y} \cos ^{2} \psi-I_{z}\right)\left(\cos ^{2} \theta-\sin { }^{2} \theta\right) \cos \phi\right] \\
& +\dot{\theta} \dot{\psi}\left\{\left(I_{y}-I_{x}\right) 2 \sin \phi \sin \psi \cos \psi+\left[\left(I_{x}-I_{y}\right)\left(\cos ^{2} \psi-\sin ^{2} \psi\right)-I_{z}\right] \cos \theta \cos \phi\right\} \\
+ & \dot{\phi} \dot{\psi} \sin \theta\left\{\left(I_{x}-I_{y}\right) 2 \cos \theta \cos \phi \sin \psi \cos ^{2}+\left[\left(I_{x}-I_{y}\right)\left(\cos ^{2} \psi-\sin ^{2} \psi\right)+I_{z}\right] \sin \phi\right\} \\
+ & \dot{\phi}^{2} \sin \theta\left[\left(I_{x}-I_{y}\right) \cos \phi \sin \psi \cos \psi+\left(I_{z}-I_{x} \sin ^{2} \psi-I_{y} \cos ^{2} \psi\right) \cos \theta \sin \phi\right] \\
+ & \dot{\theta}^{2}\left(I_{y}-I_{x}\right) \sin \theta \cos \phi \sin \psi \cos \psi,
\end{aligned}
$$




$$
\begin{aligned}
\tau_{z^{\prime}} & =\ddot{\phi}\left[\left(I_{x} \sin ^{2} \psi+I_{y} \cos ^{2} \psi\right) \sin ^{2} \theta+I_{z} \cos ^{2} \theta\right]+\ddot{\psi} I_{z} \cos \theta+\ddot{\theta}\left(I_{x}-I_{y}\right) \sin \theta \sin \psi \cos \psi \\
& +\dot{\theta} \dot{\psi} \sin \theta\left[\left(I_{x}-I_{y}\right)\left(\cos ^{2} \psi-\sin ^{2} \psi\right)-I_{z}\right]+\dot{\theta} \dot{\phi} 2 \sin \theta \cos \theta\left(I_{x} \sin ^{2} \psi+I_{y} \cos ^{2} \psi-I_{z}\right) \\
& +\dot{\phi} \dot{\psi}\left(I_{x}-I_{y}\right) 2 \sin ^{2} \theta \sin \psi \cos \psi+\dot{\theta}^{2}\left(I_{x}-I_{y}\right) \cos \theta \sin \psi \cos \psi,
\end{aligned}
$$

where $\tau_{x^{\prime}}, \tau_{y^{\prime}}$, and $\tau_{z^{\prime}}$ are components of torque in the stationary reference frame. These are the equations describing the effects of torque in the stationary reference frame in terms of Euler angles.

\subsection{Torque-angular momentum relation}

In this section, we will calculate the torque-angular momentum relation, $\vec{\tau}=d \vec{L} / d t$, by finding the time derivative of the angular momentum in the stationary reference frame.

The angular momentum can be obtained by using $\vec{L}=I \vec{w}$ as

$$
\vec{L}=\left(I_{x^{\prime} x^{\prime}} w_{x^{\prime}}+I_{x^{\prime} y^{\prime}} w_{y^{\prime}}+I_{x^{\prime} z^{\prime}} w_{z^{\prime}}\right) \hat{x}^{\prime}+\left(I_{y^{\prime} x^{\prime}} w_{x^{\prime}}+I_{y^{\prime} y^{\prime}} w_{y^{\prime}}+I_{y^{\prime} z^{\prime}} w_{z^{\prime}}\right) \hat{y}^{\prime}+\left(I_{z^{\prime} x^{\prime}} w_{x^{\prime}}+I_{z^{\prime} y^{\prime}} w_{y^{\prime}}+I_{z^{\prime} z^{\prime}} w_{z^{\prime}}\right) \hat{z}^{\prime}
$$

where $w_{i^{\prime}}$ 's are given in Eqs. (12). Here moments of inertia, i.e., Eq. (10), and inertia products, i.e., Eq. (11), are timedependent. One can find the components of $d \vec{L} / d t$ by calculating the following equations

$$
\begin{aligned}
\vec{\tau} & =\left[I_{x^{\prime} x^{\prime}} \frac{d w_{x^{\prime}}}{d t}+I_{x^{\prime} y^{\prime}} \frac{d w_{y^{\prime}}}{d t}+I_{x^{\prime} z^{\prime}} \frac{d w_{z^{\prime}}}{d t}+\frac{d I_{x^{\prime} x^{\prime}}}{d t} w_{x^{\prime}}+\frac{d I_{x^{\prime} y^{\prime}}}{d t} w_{y^{\prime}}+\frac{d I_{x^{\prime} z^{\prime}}}{d t} w_{z^{\prime}}\right] \hat{x}^{\prime} \\
& +\left[I_{y^{\prime} x^{\prime}} \frac{d w_{x^{\prime}}}{d t}+I_{y^{\prime} y^{\prime}} \frac{d w_{y^{\prime}}}{d t}+I_{y^{\prime} z^{\prime}} \frac{d w_{z^{\prime}}}{d t}+\frac{d I_{y^{\prime} x^{\prime}}}{d t} w_{x^{\prime}}+\frac{d I_{y^{\prime} y^{\prime}}}{d t} w_{y^{\prime}}+\frac{d I_{y^{\prime} z^{\prime}}}{d t} w_{z^{\prime}}\right] \hat{y}^{\prime} \\
& +\left[I_{z^{\prime} x^{\prime}} \frac{d w_{x^{\prime}}}{d t}+I_{z^{\prime} y^{\prime}} \frac{d w_{y^{\prime}}}{d t}+I_{z^{\prime} z^{\prime}} \frac{d w_{z^{\prime}}}{d t}+\frac{d I_{z^{\prime} x^{\prime}}}{d t} w_{x^{\prime}}+\frac{d I_{z^{\prime} y^{\prime}}}{d t} w_{y^{\prime}}+\frac{d I_{z^{\prime} z^{\prime}}}{d t} w_{z^{\prime}}\right] \hat{z}^{\prime} .
\end{aligned}
$$

Explicit calculations of these can be obtained as

$$
\begin{aligned}
& \tau_{x^{\prime}}=\ddot{\theta}\left[\left(I_{x} \cos ^{2} \psi+I_{y} \sin ^{2} \psi\right) \cos \phi+\left(I_{y}-I_{x}\right) \cos \theta \sin \phi \sin \psi \cos \psi\right] \\
& +\ddot{\phi} \sin \theta\left[\left(I_{x}-I_{y}\right) \cos \phi \sin \psi \cos \psi+\left(I_{z}-I_{x} \sin ^{2} \psi-I_{y} \cos ^{2} \psi\right) \cos \theta \sin \phi\right] \\
& +\ddot{\psi} I_{z} \sin \theta \sin \phi+\dot{\theta}^{2}\left(I_{x}-I_{y}\right) \sin \theta \sin \phi \sin \psi \cos \psi \\
& +\dot{\phi}^{2} \sin \theta\left[\left(I_{y}-I_{x}\right) \sin \phi \sin \psi \cos \psi+\left(I_{z}-I_{x} \sin ^{2} \psi-I_{y} \cos ^{2} \psi\right) \cos \theta \cos \phi\right] \\
& +\dot{\theta} \dot{\phi} \sin \phi\left[-I_{x} \cos ^{2} \psi-I_{y} \sin ^{2} \psi+\left(I_{z}-I_{x} \sin ^{2} \psi-I_{y} \cos ^{2} \psi\right)\left(\cos ^{2} \theta-\sin ^{2} \theta\right)\right] \\
& +\dot{\theta} \dot{\psi}\left\{\left(I_{y}-I_{x}\right) 2 \cos \phi \sin \psi \cos \psi+\left[\left(I_{x}-I_{y}\right)\left(\sin ^{2} \psi-\cos ^{2} \psi\right)+I_{z}\right] \cos \theta \sin \phi\right\} \\
& +\dot{\phi} \dot{\psi} \sin \theta\left\{\left(I_{y}-I_{x}\right) 2 \cos \theta \sin \phi \sin \psi \cos \psi+\left[\left(I_{x}-I_{y}\right)\left(\cos ^{2} \psi-\sin ^{2} \psi\right)+I_{z}\right] \cos \phi\right\}, \\
& \tau_{y^{\prime}}=\ddot{\theta}\left[\left(I_{x} \cos ^{2} \psi+I_{y} \sin ^{2} \psi\right) \sin \phi+\left(I_{x}-I_{y}\right) \cos \theta \cos \phi \sin \psi \cos \psi\right] \\
& +\ddot{\phi} \sin \theta\left[\left(I_{x}-I_{y}\right) \sin \phi \sin \psi \cos \psi+\left(I_{x} \sin ^{2} \psi+I_{y} \cos ^{2} \psi-I_{z}\right) \cos \theta \cos \phi\right] \\
& -\ddot{\psi} I_{z} \sin \theta \cos \phi+\dot{\theta}^{2}\left(I_{y}-I_{x}\right) \sin \theta \cos \phi \sin \psi \cos \psi \\
& +\dot{\phi}^{2} \sin \theta\left[\left(I_{x}-I_{y}\right) \cos \phi \sin \psi \cos \psi+\left(I_{z}-I_{x} \sin ^{2} \psi-I_{y} \cos ^{2} \psi\right) \cos \theta \sin \phi\right] \\
& +\dot{\theta} \dot{\phi} \cos \phi\left[I_{x} \cos ^{2} \psi+I_{y} \sin ^{2} \psi+\left(I_{x} \sin ^{2} \psi+I_{y} \cos ^{2} \psi-I_{z}\right)\left(\cos ^{2} \theta-\sin ^{2} \theta\right)\right] \\
& +\dot{\theta} \dot{\psi}\left\{\left(I_{y}-I_{x}\right) 2 \sin \phi \sin \psi \cos \psi+\left[\left(I_{x}-I_{y}\right)\left(\cos ^{2} \psi-\sin ^{2} \psi\right)-I_{z}\right] \cos \theta \cos \phi\right\} \\
& +\dot{\phi} \dot{\psi} \sin \theta\left\{\left(I_{x}-I_{y}\right) 2 \cos \theta \cos \phi \sin \psi \cos \psi+\left[\left(I_{x}-I_{y}\right)\left(\cos ^{2} \psi-\sin ^{2} \psi\right)+I_{z}\right] \sin \phi\right\} \text {, } \\
& \tau_{z^{\prime}}=\ddot{\theta}\left(I_{x}-I_{y}\right) \sin \theta \sin \psi \cos \psi+\ddot{\phi}\left[\left(I_{x} \sin ^{2} \psi+I_{y} \cos ^{2} \psi\right) \sin ^{2} \theta+I_{z} \cos ^{2} \theta\right] \\
& +\ddot{\psi} I_{z} \cos \theta+\dot{\theta}^{2}\left(I_{x}-I_{y}\right) \cos \theta \sin \psi \cos \psi+\dot{\phi} \dot{\psi}\left(I_{x}-I_{y}\right) 2 \sin ^{2} \theta \sin \psi \cos \psi \\
& +\dot{\theta} \dot{\psi} \sin \theta\left[\left(I_{x}-I_{y}\right)\left(\cos ^{2} \psi-\sin ^{2} \psi\right)-I_{z}\right]+\dot{\theta} \dot{\phi} 2 \sin \theta \cos \theta\left(I_{x} \sin ^{2} \psi+I_{y} \cos ^{2} \psi-I_{z}\right),
\end{aligned}
$$


where $\tau_{i}$ 's are components of torque in the stationary reference frame. It can be seen that these equations are the same as equations obtained from Euler equations in the stationary reference frame. This shows that Euler equations in the stationary reference frame and torque-angular momentum relation are equivalent, and both can be used to study rotations in the stationary reference frame.

\section{Equivalence}

In this section, we will explicitly show that Euler equations in the body reference frame are equivalent to Euler equations in the stationary reference frame.

One can obtain components of torque in the body reference frame in terms of torque components in the stationary reference frame by using the relation $\tau_{i}=\sum_{j} S_{i j}^{-1} \tau_{j^{\prime}}$ as

$$
\begin{aligned}
\tau_{x} & =\tau_{x^{\prime}}(\cos \phi \cos \psi-\cos \theta \sin \phi \sin \psi)+\tau_{y^{\prime}}(\sin \phi \cos \psi+\cos \theta \cos \phi \sin \psi)+\tau_{z^{\prime}} \sin \theta \sin \psi \\
\tau_{y} & =\tau_{x^{\prime}}(-\cos \phi \sin \psi-\cos \theta \sin \phi \cos \psi)+\tau_{y^{\prime}}(-\sin \phi \sin \psi+\cos \theta \cos \phi \cos \psi)+\tau_{z^{\prime}} \sin \theta \cos \psi \\
\tau_{z} & =\tau_{x^{\prime}} \sin \theta \sin \phi+\tau_{y^{\prime}}(-\sin \theta \cos \phi)+\tau_{z^{\prime}} \cos \theta
\end{aligned}
$$

If one multiplies Eq. (18) with $\cos \phi \cos \psi-\cos \theta \sin \phi \sin \psi$, Eq. (19) with $\sin \phi \cos \psi+\cos \theta \cos \phi \sin \psi$, Eq. (20) with $\sin \theta \sin \psi$ and add three of them, one gets

$$
\begin{aligned}
& \tau_{x^{\prime}}(\cos \phi \cos \psi-\cos \theta \sin \phi \sin \psi)+\tau_{y^{\prime}}(\sin \phi \cos \psi+\cos \theta \cos \phi \sin \psi)+\tau_{z^{\prime}} \sin \theta \sin \psi \\
& \quad=I_{x} \ddot{\phi} \sin \theta \sin \psi+I_{x} \ddot{\theta} \cos \psi+\dot{\theta} \dot{\phi} \cos \theta \sin \psi\left(I_{x}+I_{y}-I_{z}\right)+\dot{\theta} \dot{\psi} \sin \psi\left(I_{y}-I_{x}-I_{z}\right) \\
& \quad+\dot{\phi} \dot{\psi} \sin \theta \cos \psi\left(I_{x}+I_{z}-I_{y}\right)+\dot{\phi}^{2} \sin \theta \cos \theta \cos \psi\left(I_{z}-I_{y}\right)
\end{aligned}
$$

From Eq. (21), it can be seen that the right-hand side of this equation is equal to $\tau_{x}$, and then Eq. (24) is the same as the first Euler equation, i.e., Eq. (4).

Similarly, by multiplying Eq. (18) with $-\cos \phi \sin \psi-\cos \theta \sin \phi \cos \psi$, Eq. (19) with $-\sin \phi \sin \psi+\cos \theta \cos \phi \cos \psi$, Eq. (20) with $\sin \theta \cos \psi$ and adding them, one gets

$$
\begin{aligned}
& -\tau_{x^{\prime}}(\cos \phi \sin \psi+\cos \theta \sin \phi \cos \psi)-\tau_{y^{\prime}}(\sin \phi \sin \psi-\cos \theta \cos \phi \cos \psi)+\tau_{z^{\prime}} \sin \theta \cos \psi \\
& =I_{y} \ddot{\phi} \sin \theta \cos \psi-I_{y} \ddot{\theta} \sin \psi+\dot{\theta} \dot{\phi} \cos \theta \cos \psi\left(I_{x}+I_{y}-I_{z}\right)+\dot{\theta} \dot{\psi} \cos \psi\left(I_{x}-I_{y}-I_{z}\right) \\
& +\dot{\phi} \dot{\psi} \sin \theta \sin \psi\left(I_{x}-I_{y}-I_{z}\right)+\dot{\phi}^{2} \sin \theta \cos \theta \sin \psi\left(I_{x}-I_{z}\right) .
\end{aligned}
$$

From Eq. (22), it can be seen that Eq. (25) is the same as the second Euler equation, i.e., Eq. (5).

By multiplying Eq. (18) with $\sin \theta \sin \phi$, Eq. (19) with $-\sin \theta \cos \phi$, Eq. (20) with $\cos \theta$ and adding them, one obtains

$$
\begin{aligned}
\tau_{x^{\prime}} \sin \theta \sin \phi & -\tau_{y^{\prime}} \sin \theta \cos \phi+\tau_{z^{\prime}} \cos \theta=I_{z} \ddot{\phi} \cos \theta+I_{z} \ddot{\psi}+\dot{\theta} \dot{\phi} \sin \theta\left[\left(I_{y}-I_{x}\right)\left(\cos ^{2} \psi-\sin ^{2} \psi\right)-I_{z}\right] \\
& +\dot{\theta}^{2} \sin \psi \cos \psi\left(I_{x}-I_{y}\right)+\dot{\phi}^{2} \sin ^{2} \theta \sin \psi \cos \psi\left(I_{y}-I_{x}\right) .
\end{aligned}
$$

From Eq. (23), it can be seen that Eq. (26) is the same as the third Euler equation, i.e., Eq. (6).

These calculations also show that Euler equations are equivalent to torque-angular momentum relation since components of torque angular momentum relation are the same as Euler equations in the stationary reference frame.

\section{Summary and Conclusion}

We have considered a rigid body having different moments of inertia. Then we have calculated Euler equations in the body and stationary reference frames, and we have also calculated torque-angular momentum relation in terms of Euler angles and their time derivatives. Then, we have shown that Euler equations in both reference frames and $\vec{\tau}=d \vec{L} / d t$ give the same results. We have also shown in the appendix that the same equations can be obtained from Lagrangian as expected.

The equivalence of Euler equations in both reference frames shows that it is possible to study rigid body rotations in the stationary reference frame. However, it is better to use Euler equations in the body reference frame due to non-zero inertia products and the time-dependent structure of moments of inertia and inertia products in the stationary reference frame.

The equivalence of Euler equations and torque-angular momentum relation shows that extra terms in the Euler equations or gyroscopic effects are nothing but results of the change in angular momentum. 


\section{Appendix A.}

Here, we will obtain equations of motion for a rotating rigid body from the Lagrangian in the body reference frame. In the body reference frame, kinetic energy can be written as

$$
T=\frac{1}{2} I_{x} w_{x}^{2}+\frac{1}{2} I_{y} w_{y}^{2}+\frac{1}{2} I_{z} w_{z}^{2},
$$

where $w_{i}$ 's are given in Eqs. (3). We can consider a potential energy $U(\theta, \phi, \psi)$, which satisfies $\tau_{\theta}=-\partial U / \partial \theta, \tau_{\phi}=-\partial U / \partial \phi$ and $\tau_{\psi}=-\partial U / \partial \psi$. Then from Lagrangian $L=T-U$, Euler-Lagrange equations, $d / d t\left(\partial L / \partial \dot{q}_{i}\right)-\partial L / \partial q_{i}=0$, can be calculated. The Euler-Lagrange equation for $\theta$ can be obtained as

$$
\begin{aligned}
\tau_{\theta} & =\ddot{\theta}\left(I_{x} \cos ^{2} \psi+I_{y} \sin ^{2} \psi\right)+\ddot{\phi}\left(I_{x}-I_{y}\right) \sin \theta \sin \psi \cos \psi \\
& +\dot{\theta} \dot{\psi}\left(I_{y}-I_{x}\right) 2 \sin \psi \cos \psi+\dot{\phi} \dot{\psi} \sin \theta\left[\left(I_{x}-I_{y}\right)\left(\cos ^{2} \psi-\sin ^{2} \psi\right)+I_{z}\right] \\
& +\dot{\phi}^{2} \sin \theta \cos \theta\left(I_{z}-I_{x} \sin ^{2} \psi-I_{y} \cos ^{2} \psi\right),
\end{aligned}
$$

where $\tau_{\theta}$ is equal to $\tau_{x^{\prime}} \cos \phi+\tau_{y^{\prime}} \sin \phi$. If we multiply Eq. (18) with $\cos \phi$, Eq. (19) with $\sin \phi$ and add them, we obtain Eq. (A.2).

The Euler-Lagrange equation for $\phi$ can be obtained as

$$
\begin{aligned}
\tau_{\phi} & =\ddot{\theta}\left(I_{x}-I_{y}\right) \sin \theta \sin \psi \cos \psi+\ddot{\psi} I_{z} \cos \theta+\ddot{\phi}\left[\left(I_{x} \sin ^{2} \psi+I_{y} \cos ^{2} \psi\right) \sin ^{2} \theta+I_{z} \cos ^{2} \theta\right] \\
& +\dot{\theta}^{2}\left(I_{x}-I_{y}\right) \cos \theta \sin \psi \cos \psi+\dot{\theta} \dot{\psi} \sin \theta\left[\left(I_{x}-I_{y}\right)\left(\cos ^{2} \psi-\sin ^{2} \psi\right)-I_{z}\right] \\
& +\dot{\theta} \dot{\phi} 2 \sin \theta \cos \theta\left[I_{x} \sin ^{2} \psi+I_{y} \cos ^{2} \psi-I_{z}\right]+\dot{\phi} \dot{\psi}\left(I_{x}-I_{y}\right) 2 \sin ^{2} \theta \sin \psi \cos \psi .
\end{aligned}
$$

$\tau_{\phi}$ is equal to $\tau_{z^{\prime}}$ and then this equation is the same as Eq. (20).

The Euler-Lagrange equation for $\psi$ can be obtained as

$$
\begin{aligned}
\tau_{\psi} & =\ddot{\psi} I_{z}+\ddot{\phi} I_{z} \cos \theta+\dot{\theta}^{2}\left(I_{x}-I_{y}\right) \sin \psi \cos \psi \\
& +\dot{\phi}^{2}\left(I_{y}-I_{x}\right) \sin ^{2} \theta \sin \psi \cos \psi+\dot{\theta} \dot{\phi} \sin \theta\left[\left(I_{x}-I_{y}\right)\left(\sin ^{2} \psi-\cos ^{2} \psi\right)-I_{z}\right] .
\end{aligned}
$$

$\tau_{\psi}$ is equal to $\tau_{z}$, and then this equation is the same as Eq. (6).

Since Euler equations in the stationary reference frame and the body reference frame are equivalent, equations obtained from Lagrangian are also equivalent to them.

1. L. Euler, Decouverte d'un noveau principe de mecanique, The Euler Archive E-177 (1752), http: / / eulerarchive. maa.org

2. L. Euler, Du mouvement de rotation des corps solides autour d'un axe variable, The Euler Archive E-292 (1765), http: //eulerarchive.maa.org

3. J. E. Marquina, M. L. Marquina, V. Marquina and J. J. Hernández-Gómez, Leonhard Euler and the mechanics of rigid bodies, Eur. J. Phys., 38 (2017) 015001, https://doi. org/10.1088/0143-0807/38/1/015001

4. V. Tanriverdi, Comment on 'Leonhard Euler and the mechanics of rigid bodies', Submitted to Eur. J. Phys, (2020)

5. H. Goldstein, Classical Mechanics, 2nd ed. (Massachusetts: Addison-Wesley, Massachusetts, 1980)

6. J. B. Marion and S. T. Thornton, Classical Dynamics of Particles and Systems, 5th ed. (Brooks/Cole, Belmont, 2004)

7. A. Sommerfeld, Mechanics Lectures on Theoretical Physics. Volume 1 (Academic Press, New York, 1952)
8. J. R. Taylor, Classical Mechanics, (Dulles: University Science Books, Dulles, 2005)

9. G. R. Fowles and G. L. Cassiday, Analytical Mechanics, 7th ed. (Brooks/Cole, Belmont, 2005)

10. V. Barger and M. Olson, Classical Mechanics: A Modern Perspective, (McGraw-Hill, New York, 1994)

11. V. J. Jorge and J. S. Eugene, Classical dynamics. A contemporary approach, (Cambridge University Press, Cambridge, 1998)

12. J. L. McCauley, Classical mechanics: Transformations, flows, integrable, and chaotic dynamics, (Cambridge: Cambridge University Press, Cambridge, 1997)

13. E. Corinaldesi, Classical Mechanics for Physics Graduate Students, (World Scientific Publishing Company, Singapore, 1999)

14. E. A. Desloge, Classical Mechanics. Volume 1, (New York: John Wiley and Sons, New York, 1982) 
15. K. R. Symon, Mechanics, 3rd ed. (Addison-Wesley, Massachusetts, 1971)

16. R. D. Gregory, Classical Mechanics, (Cambridge University Press, New York, 2006)

17. V. I. Arnold, Mathematical Methods of Classical Mechanics, 2nd ed. (Springer-Verlag, New York, 1989)

18. L. D. Landau and E. M. Lifshitz, Mechanics, 3rd ed. (Butterworth-Heinenann, New Delhi, 2000)

19. W. D. MacMillan, Dynamics Of Rigid Bodies, (Dover, New York, 1960)

20. W. Greiner, Classical Mechanics, Systems of particles and Hamiltonian dynamics, (Springer, New York, 2003)

21. J. B. Scarborough, The Gyroscope Theory and Applications, (Interscience Publishers, London, 1958)

22. E. Leimanis, The General Problem of the Motion of Coupled Rigid Bodies about a Fixed Point, (Springer-Verlag, Berlin, 1965)
23. L. D. Mott, Another Derivation of Euler's Equations of RigidBody Rotation, Am. J. Phys. 34 (1966) 1197, https : / / doi. org/10.1119/1.1972684

24. H. Lamb, Higher Mechanics, (Cambridge University Press, Cambridge, 1920)

25. H. Crabtree, An elementary treatment of the theory of spinning tops and gyroscopic motion, (Longmans, Green and Co., London, 1909)

26. R. B. Hayward, On a direct method of estimating velocities, accelerations, and all similar quantities with respect to axes moveable in any manner in space, with applications, Cambridge Philosophical Society. Transactions of the Cambridge Philosophical Society (Cambridge: University Press), 10 (1864) 1-20, https://catalog.hathitrust.org/Record/ 000526741

27. F. Klein and A. Sommerfeld, The theory of the Top, Volume II, (Birkhauser, Boston MA, 2010) 